\title{
MIRADAS for the Gran Telescopio Canarias
}

Stephen S. Eikenberry, Steven Nicholas Raines, Richard Deno Stelter, Alan Garner, Yigit Dallilar, Kendall Ackley, John Greg Bennett, Charles H. Murphey, Paola Miller, David Tooke, Luke Williams, Brian Chinn, Scott A. Mullin, Sidney L. Schofield, Craig D. Warner, Frank Varosi, Bo

Zhao, Sophia A. Eikenberry, Claudia Vega, Hipatia V. Donoso

Department of Astronomy, University of Florida, Gainesville, FL 32611

Josep Sabater, José María Gómez, Jordi Torra

Universidad de Barcelona, Barcelona, Spain

Josefina Rosich Minguell, Francisco Garzón López, Pablo López Ramos

Instituto Astrofisica de Canarias, La Laguna, Tenerife, Spain

Nicolás Cardiel, Jesús Gallego Maestro

Universidad Complutense de Madrid, Madrid, Spain

Antonio Marín-Franch

CEFCA, Teruel, Spain

Julio Galipienzo, Miguel Ángel Carrera Astigarraga

Added Value Solutions, Eibar, Spain

Greg J. Fitzgerald, Ian Prees, Todd M. Stolberg, Peter A. Kornik

New England Optical Systems, Marlborough, MA

Anamparambu N. Ramaprakash, Mahesh P. Burse, Sujit P. Punnadi

IUCAA, Pune, India

P. Hammersley

European Southern Observatory, Garching, Germany

\begin{abstract}
The Mid-resolution InfRAreD Astronomical Spectrograph (MIRADAS, a near-infrared multi-object echelle spectrograph operating at spectral resolution $\mathrm{R}=20,000$ over the 1-2.5 $\mu \mathrm{m}$ bandpass) was selected by the Gran Telescopio Canarias (GTC) partnership as the next-generation near-infrared spectrograph for the world's largest optical/infrared telescope, and is being developed by an international consortium. The MIRADAS consortium includes the University of Florida, Universidad de Barcelona, Universidad Complutense de Madrid, Instituto de Astrofísica de Canarias, and Institut d'Estudis Espacials de Catalunya, as well as probe arm industrial partner A-V-S (Spain), with more than 45 Science Working Group members in 10 institutions primarily in Spain, Mexico, and the USA. In this paper, we review the overall system design and project status for MIRADAS during its early fabrication phase in 2016.
\end{abstract}

Ground-based and Airborne Instrumentation for Astronomy VI, edited by Christopher J. Evans, Luc Simard, Hideki Takami Proc. of SPIE Vol. 9908, 99081L · (c) 2016 SPIE · CCC code: 0277-786X/16/\$18 · doi: 10.1117/12.2232533 


\section{INTRODUCTION}

The Mid-resolution InfRAreD Astronomical Spectrograph (MIRADAS) for GTC is the most powerful astronomical instrument of its kind ever developed. The combination of the collecting area of GTC and the multi-object midresolution near-infrared spectra provided by MIRADAS make its capabilities unparalleled for addressing some of the leading scientific challenges of the coming decades. The MIRADAS Science Working Group has identified 4 key science cases for MIRADAS. These 4 key cases form the "Design Reference Cases" for MIRADAS - the scientific performance capabilities which provide the fundamental requirements drivers for the instrument. In this paper, we present the basic instrument overview, which covers the basic concept of the instrument intended to meet the requirements of the Design Reference Cases.

The basic MIRADAS concept (Eikenberry et al., 2012) is a near-infrared multi-object echelle spectrograph operating at spectral resolution $\mathrm{R}=20,000$ over the 1-2.5 $\mu \mathrm{m}$ bandpass. MIRADAS selects targets using up to 12 deployable probe arms with pickoff mirror optics, each feeding a 3.7x1.2-arcsec field of view to the spectrograph. The spectrograph input optics also include a "slit slicer" which reformats each probe field into 3 end-to-end slices of a fixed 3.7x0.4-arcsec format - combining the advantages of minimal slit losses in any seeing conditions better than 1.2-arcsec, while at the same time providing some (limited) two-dimensional spatial resolution. The spectrograph optics then provide a range of configurations providing the observer with the ability to choose between maximal multiplex advantage and maximal wavelength coverage, with several intermediate options, depending upon the needs of the science program. Its basic parameters are as follows:

Table 1 - General Parameters

\begin{tabular}{|l|c|l|}
\hline Parameter & Value & Comment \\
\hline Target field of regard & 5-arcminute diameter & $\begin{array}{l}\text { Each probe arm patrols a "slice of } \\
\text { pie" wedge of this circular field }\end{array}$ \\
\hline Individual target field of view & $3.7 \times 1.2-$ arcsec & \\
\hline Slit slicer geometry & 3 slices of 3.7x0.4-arcsec ea. & \\
\hline Detector focal plane & Linear, circular & $\begin{array}{l}\text { Available for single-object cross- } \\
\text { dispersed mode }\end{array}$ \\
\hline Spectro-polarimetry & $\begin{array}{l}\mathrm{J}=18.0 \mathrm{mag} \\
\mathrm{H}=17.4 \mathrm{mag} \\
\mathrm{K}=16.7 \mathrm{mag}\end{array}$ & $\begin{array}{l}\mathrm{S} / \mathrm{N}=10 \text { for 1-hour on-source } \\
\text { exposure }\end{array}$ \\
\hline Continuum sensitivity & $5 \times 10^{-18} \mathrm{ergs} / \mathrm{cm}^{2} / \mathrm{s}$ (point) & $\begin{array}{l}\mathrm{S} / \mathrm{N}=10 \text { for 1-hour on-source } \\
\text { exposure; resolved source } \\
\text { assumes 1 square arcsecond } \\
\text { detect cell }\end{array}$ \\
\hline Emission line sensitivity & $8 \times 10^{-18} \mathrm{ergs} / \mathrm{cm}^{2} / \mathrm{s}$ (resolved) & \\
\hline
\end{tabular}

Table 2 - Multiplex Configurations

\begin{tabular}{|l|c|l|}
\hline \multicolumn{1}{|c|}{ Configuration } & Number of MXS Targets & \multicolumn{1}{c|}{ Instantaneous Bandpass } \\
\hline SO-mode short & 1 & $1.04-1.78 \mu \mathrm{m}$ \\
\hline SO-mode long & 1 & $1.34-2.50 \mu \mathrm{m}$ \\
\hline Medium MXS J & 3 & $1.10-1.40 \mu \mathrm{m}$ \\
\hline Medium MXS H & 3 & $1.50-1.80 \mu \mathrm{m}$ \\
\hline Medium MXS K & 2 & $1.98-2.50 \mu \mathrm{m}$ \\
\hline Maximum MXS & 12 & Any SINGLE order from Table 3 \\
\hline
\end{tabular}




\section{Table 3 - MIRADAS Echelle Orders}

\begin{tabular}{|c|c|c|c|c|c|c|}
\hline Order & $\begin{array}{c}\text { Wavelength } \\
(\boldsymbol{\mu m})\end{array}$ & Band & & Order & $\begin{array}{c}\text { Wavelength } \\
(\boldsymbol{\mu m})\end{array}$ & Band \\
\hline 14 & $2.370-2.500$ & $\mathrm{~K}$ & & 24 & $1.413-1.472$ & - \\
\hline 15 & $2.222-2.382$ & $\mathrm{~K}$ & & 25 & $1.356-1.410$ & $\mathrm{~J}$ \\
\hline 16 & $2.089-2.225$ & $\mathrm{~K}$ & & 26 & $1.311-1.349$ & $\mathrm{~J}$ \\
\hline 17 & $1.936-2.086$ & $\mathrm{~K}$ & & 27 & $1.260-1.299$ & $\mathrm{~J}$ \\
\hline 18 & $1.870-1.970$ & - & & 28 & $1.217-1.253$ & $\mathrm{~J}$ \\
\hline 19 & $1.787-1.853$ & $\mathrm{H}$ & & 29 & $1.175-1.210$ & $\mathrm{~J}$ \\
\hline 20 & $1.694-1.761$ & $\mathrm{H}$ & & 30 & $1.136-1.170$ & - \\
\hline 21 & $1.614-1.681$ & $\mathrm{H}$ & & 31 & $1.101-1.129$ & $\mathrm{~J}-l o$ \\
\hline 22 & $1.541-1.604$ & $\mathrm{H}$ & & 32 & $1.066-1.109$ & $\mathrm{~J}-l o$ \\
\hline 23 & $1.475-1.536$ & - & & 33 & $1.034-1.061$ & $\mathrm{~J}-l o$ \\
\hline & & & & 34 & $1.005-1.030$ & $\mathrm{~J}-l o$ \\
\hline
\end{tabular}

We present a conceptual layout (“cartoon” sketch) for MIRADAS in Figure 1, and the resulting scientific grasp comparison for the instrument in Figure 2. In Figure 3, we show the evolution of the MIRADAS focal planes. 


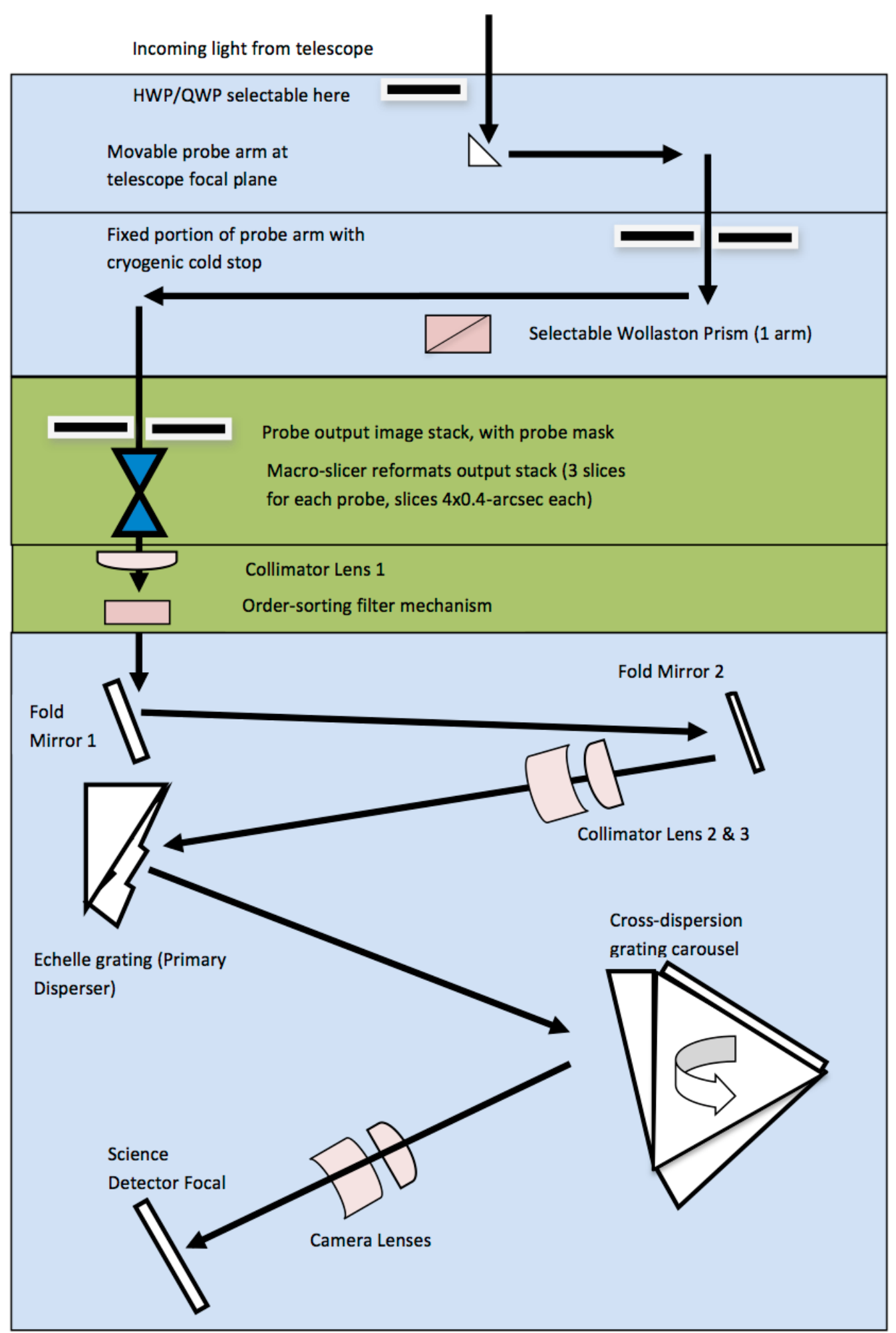

Figure 1 - MIRADAS Conceptual Layout Schematic 


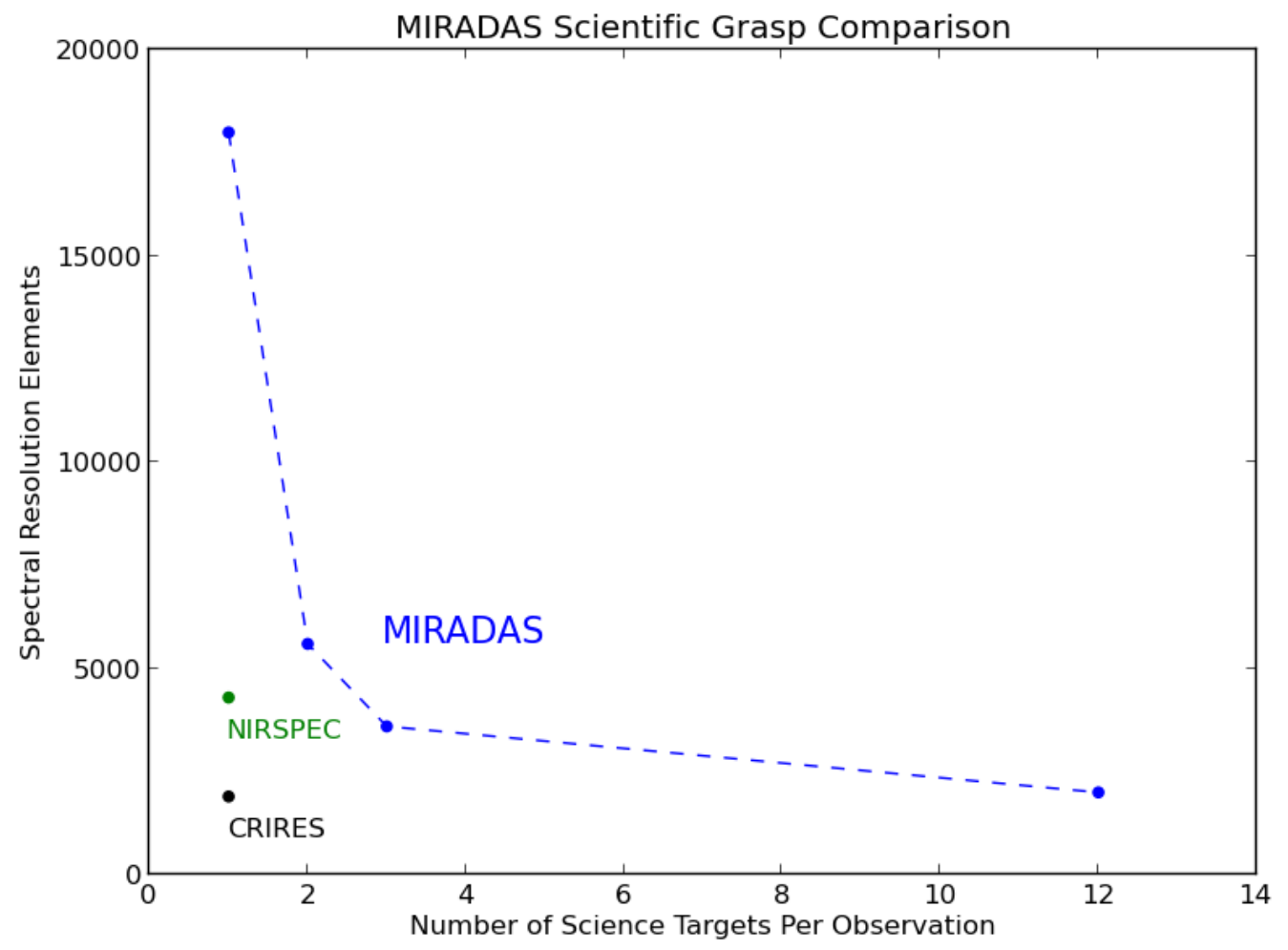

Figure 2: MIRADAS scientific grasp (instantaneous number of targets and spectral resolution elements per target) compared to other 8-m-class spectrographs with $R=20,000$ or higher. Note that MIRADAS is the only multi-object spectrograph in this class, and it provides more spectral information for each of its $N=2$ targets than others do for $N=1$ target. 

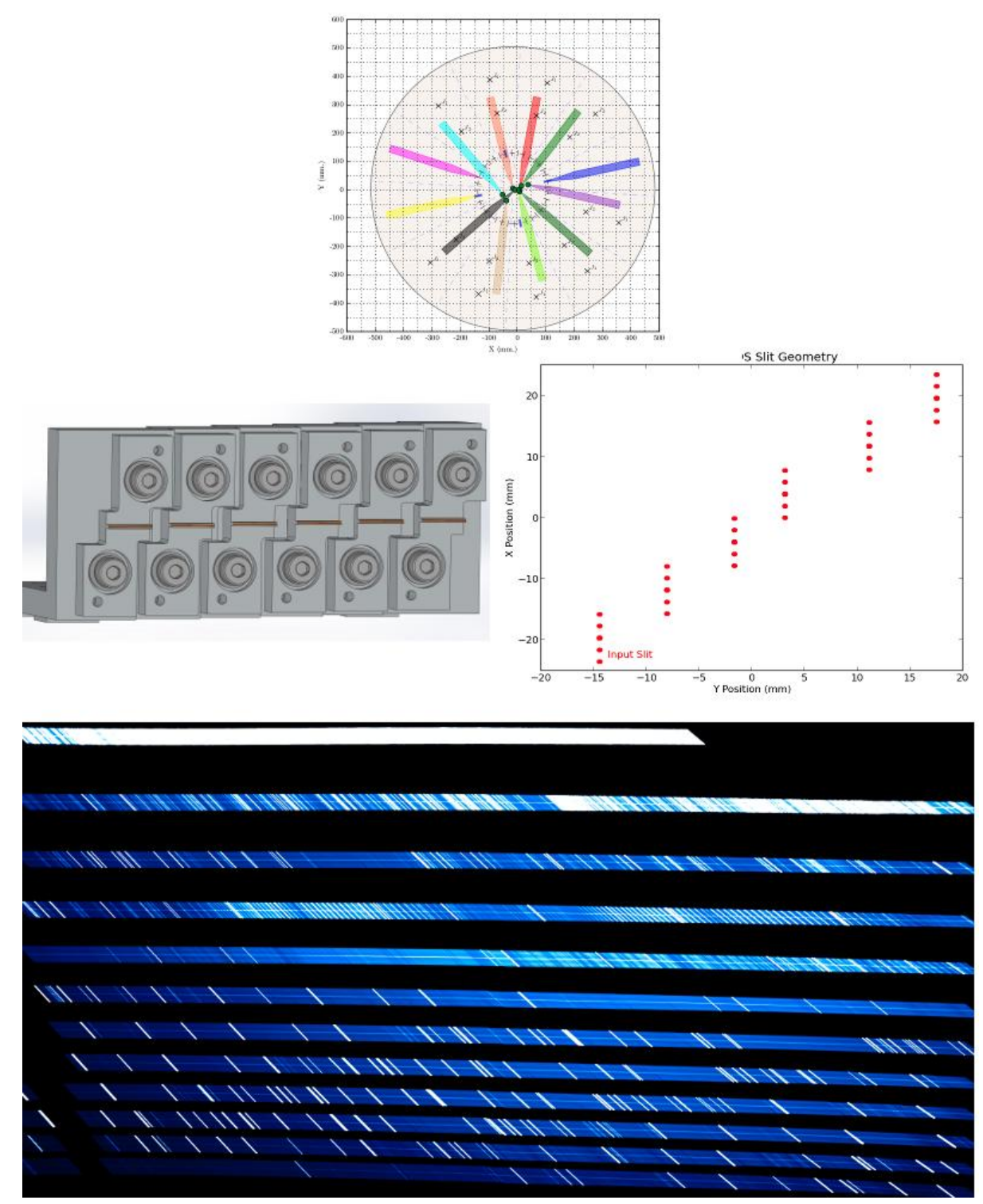

Figure 3: MIRADAS focal plane evolution. (Top) Schematic view of MXS probe arms selecting targets within the 5-arcmin field of regard at the GTC focal plane; (Middle-left) View of the slicer stack at the input to the macro-slicer; (Middle-right) Schematic view of the "sliced" probe outputs forming the pseudo-longslit at the third focal plane (spectrograph input slit); (Bottom) Cross-dispersed Single-Object Long spectrum at the final focal plane (science detectors).

\section{KEY TECHNOLOGIES: PROBE ARMS}

MIRADAS includes a multiplexing system (MXS) employing up to 12 deployable probe arms with pickoff mirrors (Eikenberry et al., 2014). MIRADAS MXS probe arms are based on previous conceptual designs for HRNIRS (Muller et al., 2006; Eikenberry et al., 2006a; Hinkle et al., 2006; Liang et al., 2006) and IRMOS (Eikenberry et al., 2006b; Andersen at al., 2006) for the Gemini Observatories.

The MXS patrol field for MIRADAS is 5-arcminutes in diameter, or about 250-mm. The primary function of the MXS probe mechanism is to select target fields-of-view and relay light from them to the rest of the spectrograph. This is accomplished using 12 independently-controlled/actuated MXS pick-off probe arms. Each arm patrols a sector of the MIRADAS field, just below the input telescope focal plane (see Sabater et al., 2016 and Sabater et al., 2014 for details on the patrol scheme and control algorithms). Optics in each arm relay light from a 3.7x1.2-arcsec field. Figure 4 
shows a view of the MXS probe mechanism. A pickoff mirror located near the telescope focal plane relays light down the probe arm, where it encounters a collimating doublet lens. The lens feeds light through a series of folds in the probe mechanism, which maintain a fixed optical path length while the probe arm is moved to variable target locations in the field of regard. The $4^{\text {th }}$ fold mirror is under the MXS optical bench, and is fixed in its location and orientation. Shortly after the fixed fold comes the cold pupil stop, located at the pupil image created by the collimator doublet. Finally, a reimaging doublet brings the beam to a focus.
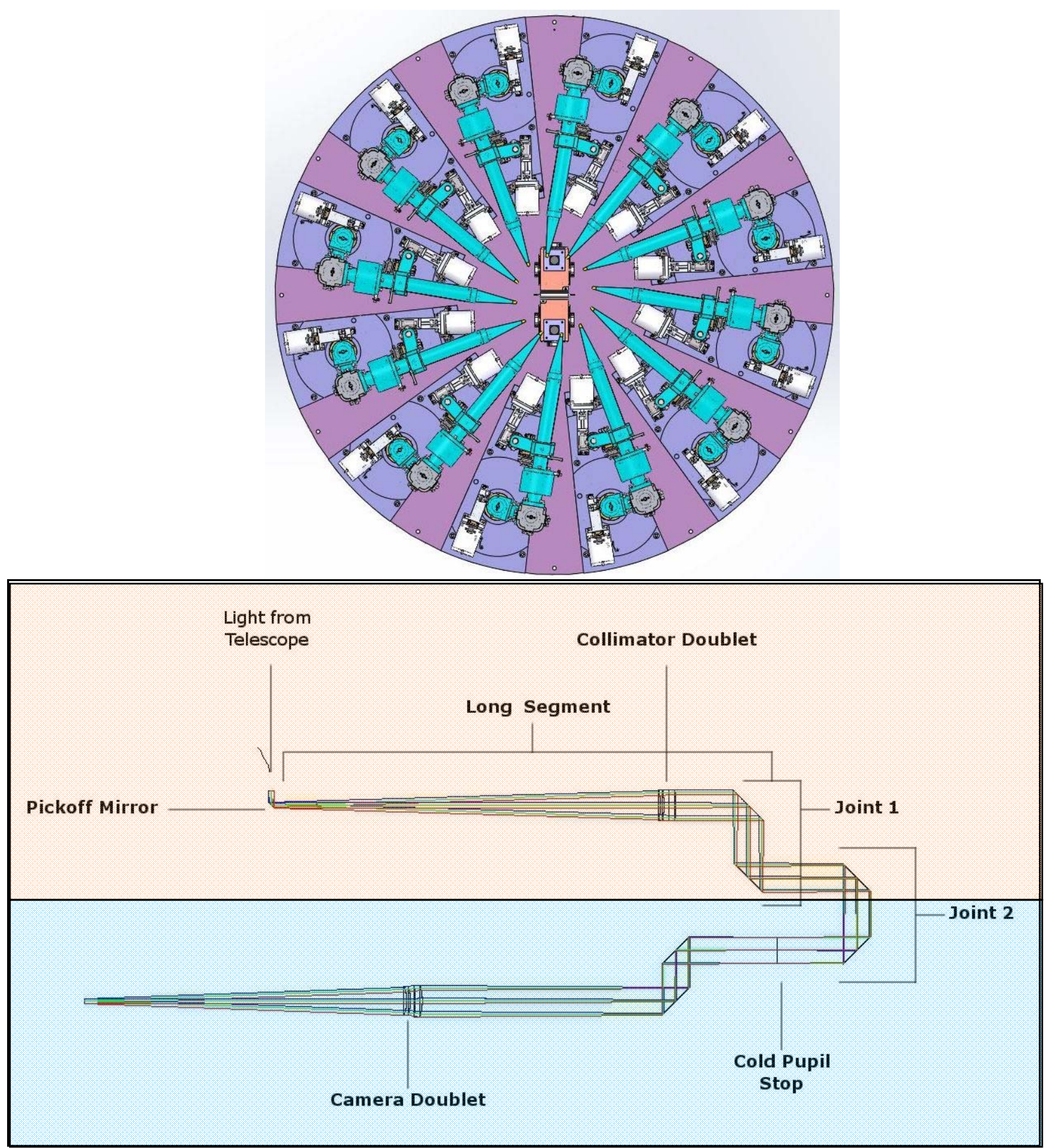

Figure 4 - MIRADAS MXS probe optical layout. The top (pink) shaded portion includes the moving parts of the probe arm, while the bottom (blue) shaded portion includes the fixed parts of the probe arm optical train. 
At the output focal plane from the MXS system, the individual probes produce fields of 3.7.0x1.2-arcsec (2.7x0.8-mm), each of which is separated from the next probe by a 10.3-mm center-to-center distance, creating a sparsely-filled pseudolongslit at the macro-slicer input.

In Figure 5, we present a more detailed view of a single probe arm mechanical model, as well as a cryogenic prototype probe arm (P3) constructed and tested at UF in 2014-2015.
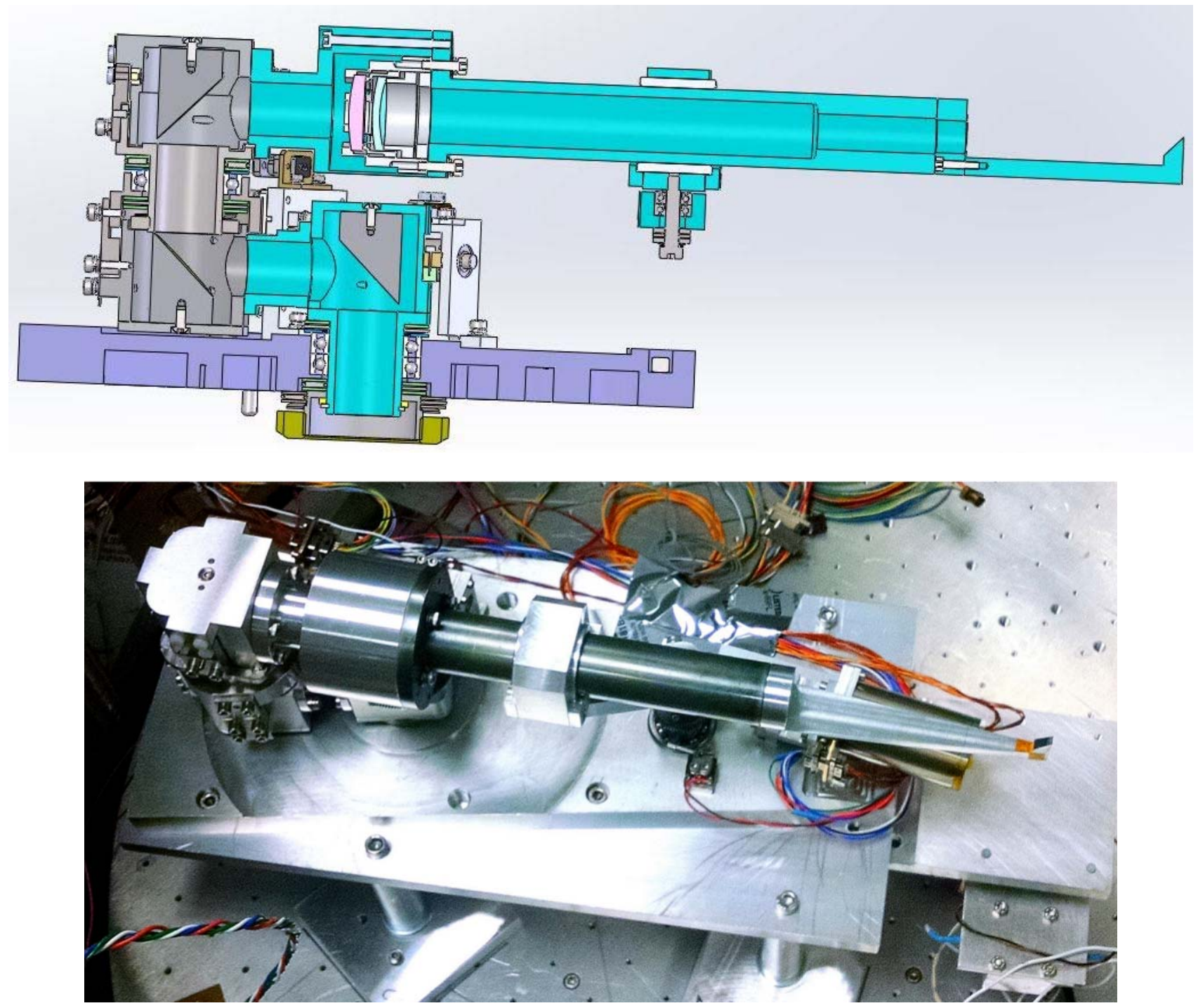

Figure 5 - (Top) Detailed section view of a single MIRADAS probe arm. (Bottom) Photo of cryogenic Prototype 3 at the University of Florida. 

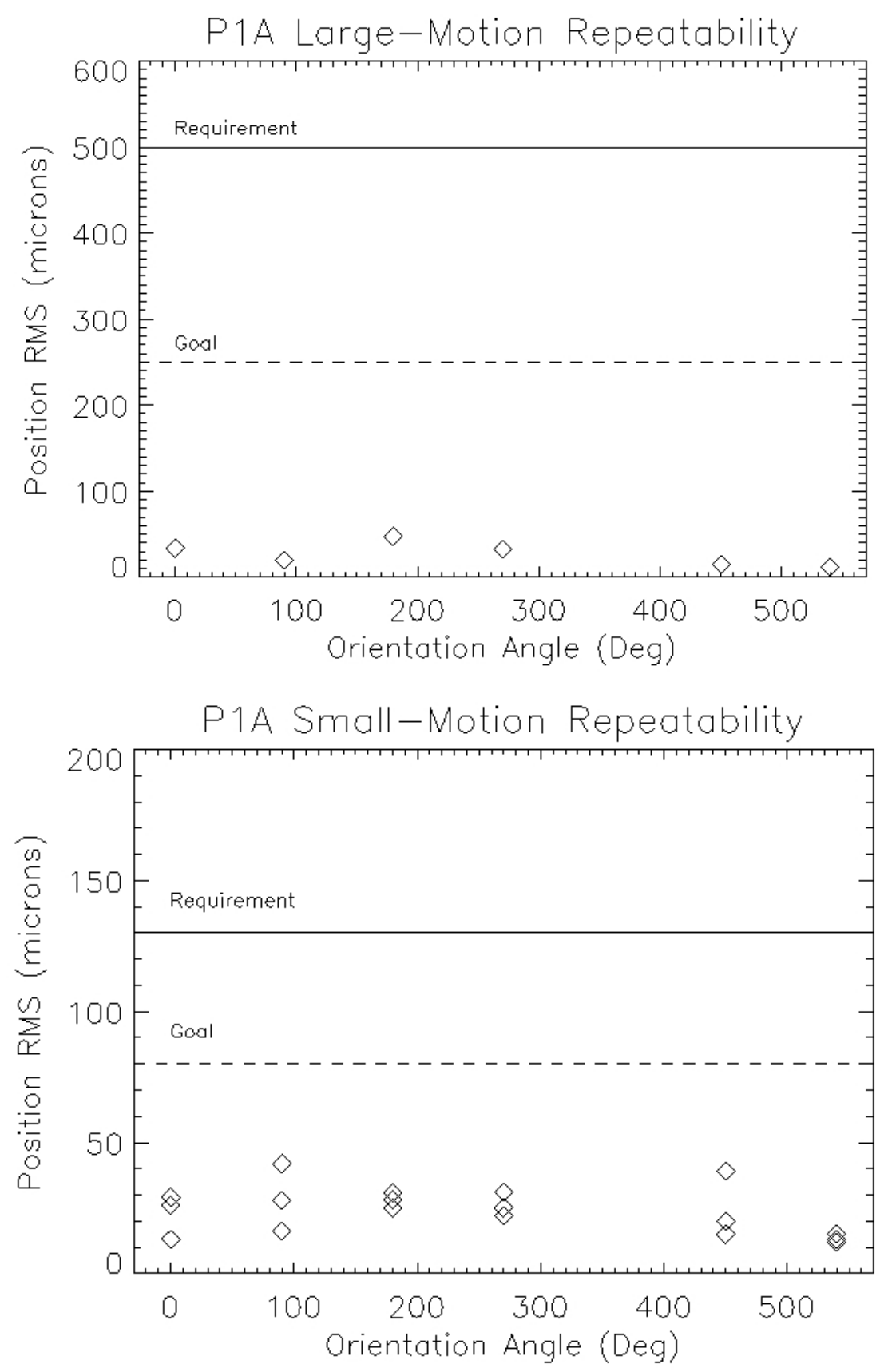

Figure 6 - Measured repeatability performance of probe arm motions for the Prototype 1 A probe arm. Note that the arm RMS is MUCH better than the requirement and goal performance established for both large and small motions. The cryogenic prototype arms (P2 and P3) both show similar performance

\section{KEY TECHNOLOGIES: MACRO-SLICER}

The next key component for MIRADAS is the "macro-slicer". This is an image slicing mini-IFU which takes the input focal plane (a 200x0.8-mm sparsely-filled pseudo-longslit) and slices it into a different, nearly-filled, pseudo-longslit of length $~ 80.5 \times 0.135-\mathrm{mm}$ (i.e. dividing each slitlet into 3 narrower slitlets oriented lengthwise). This is the same approached used for the FISICA and FRIDA image slicers. 
The input focal plane of the MIRADAS macro-slicer differs significantly from that of the previous slicer IFUs such as FISICA and FRIDA - not only in it narrow aspect ratio, but also in the length of the field ( 200-mm versus $\sim 20-30$-mm for FISICA/FRIDA). For typical previous slicers, the curvature of the slicer mirror itself would introduce significant defocus issues over such a long distance. However, the MIRADAS macro-slicer input also differs from the previous IFUs in that its input field is NOT a continuous telescopic focal image, but is in fact a sparsely-filled field composed of disjoint relay inputs. As such, we can freely "segment" the slicer array into sub-slicers - each with the required width, but much shorter length (thus reducing the mirror curvature defocus issue) and with the between-segment gaps chosen to lie between the disjoint probe fields. We thus separate the slicer mirror into 5 slicer segments, each of which covers 4 MXS probe inputs. The slicer length is thus reduced to $35-\mathrm{mm}$ - very similar to the values used for FRIDA and FISICA, and thus reducing the curvature problem to being nearly negligible.

From that point on, the macro-slicer more closely resembles the previous slicer IFUs built at the University of Florida, with each slice being mapped to a pupil mirror in an array, and from there focused onto an output focal plane in the form of a nearly-filled pseudo-longslit. Details of this subsystem are presented by Stelter \& Eikenberry (these proceedings).

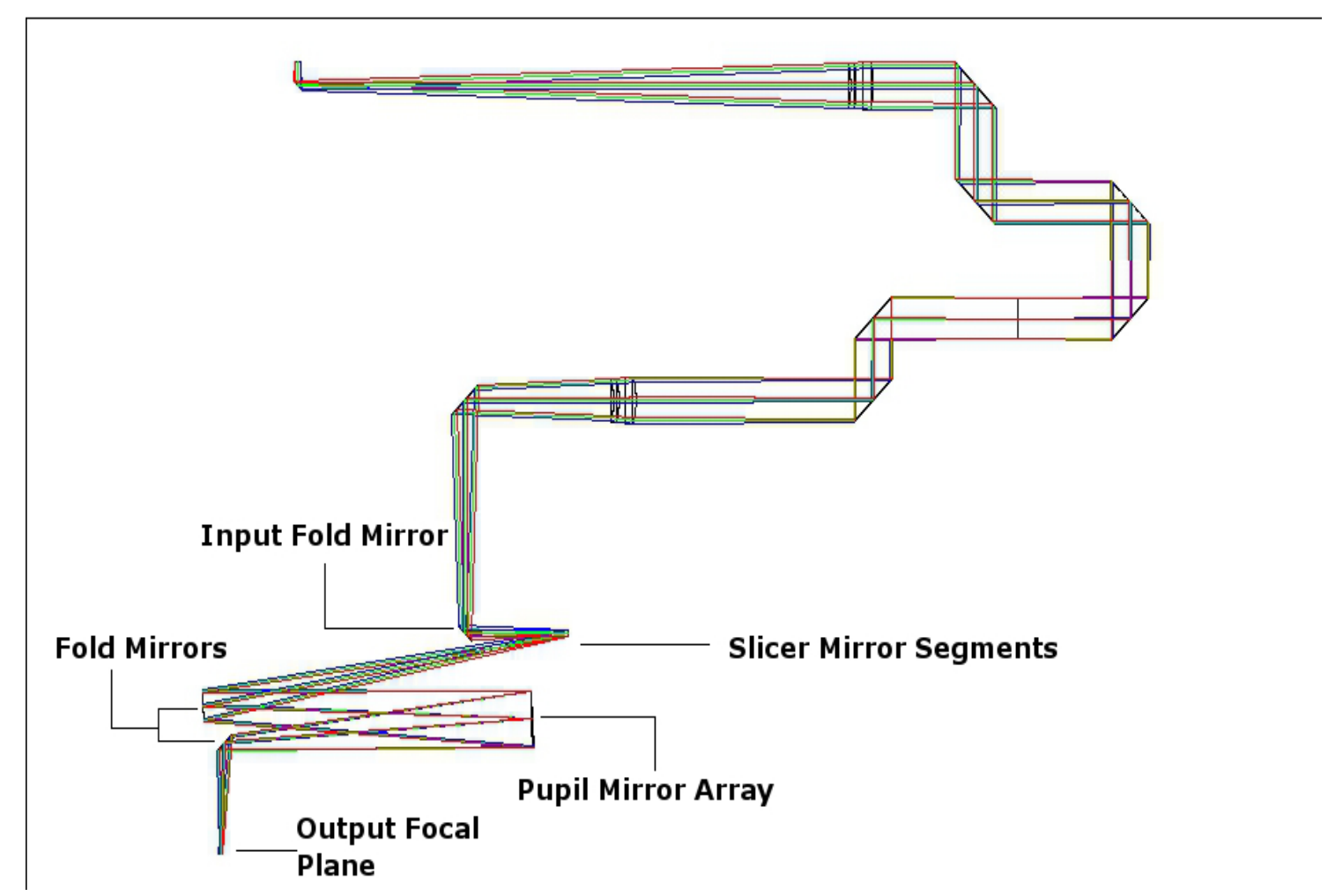

Figure 7 - Probe arm and macro-slicer ZEMAX layout

\section{SPECTRAL MODES AND FORMATS}

For the various modes described above, MIRADAS provides different spectral layouts and formats on the 4096x2048pixel detector mosaic. We present examples of those modes $(\mathrm{N}=1, \mathrm{~N}=3, \mathrm{~N}=12)$ those graphically here. 


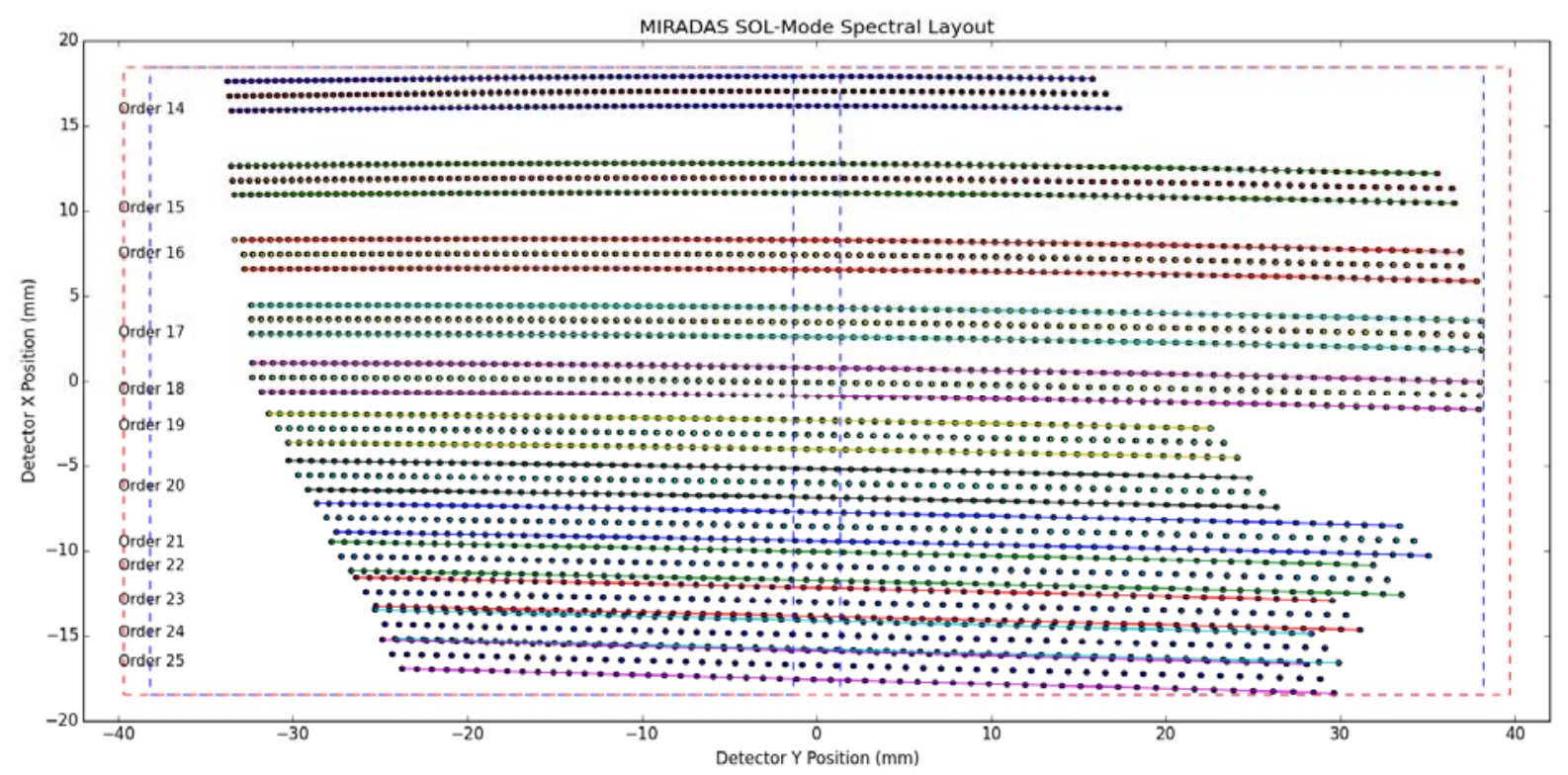

Figure 8 - MIRADAS single-object long-wavelength mode cross-dispersed spectrum projected on the 4096x2048-pixel detector mosaic. The spectrum covers the wavelength range from 1.34-2.50 $\mu$ m (orders 14-25 from top to bottom).

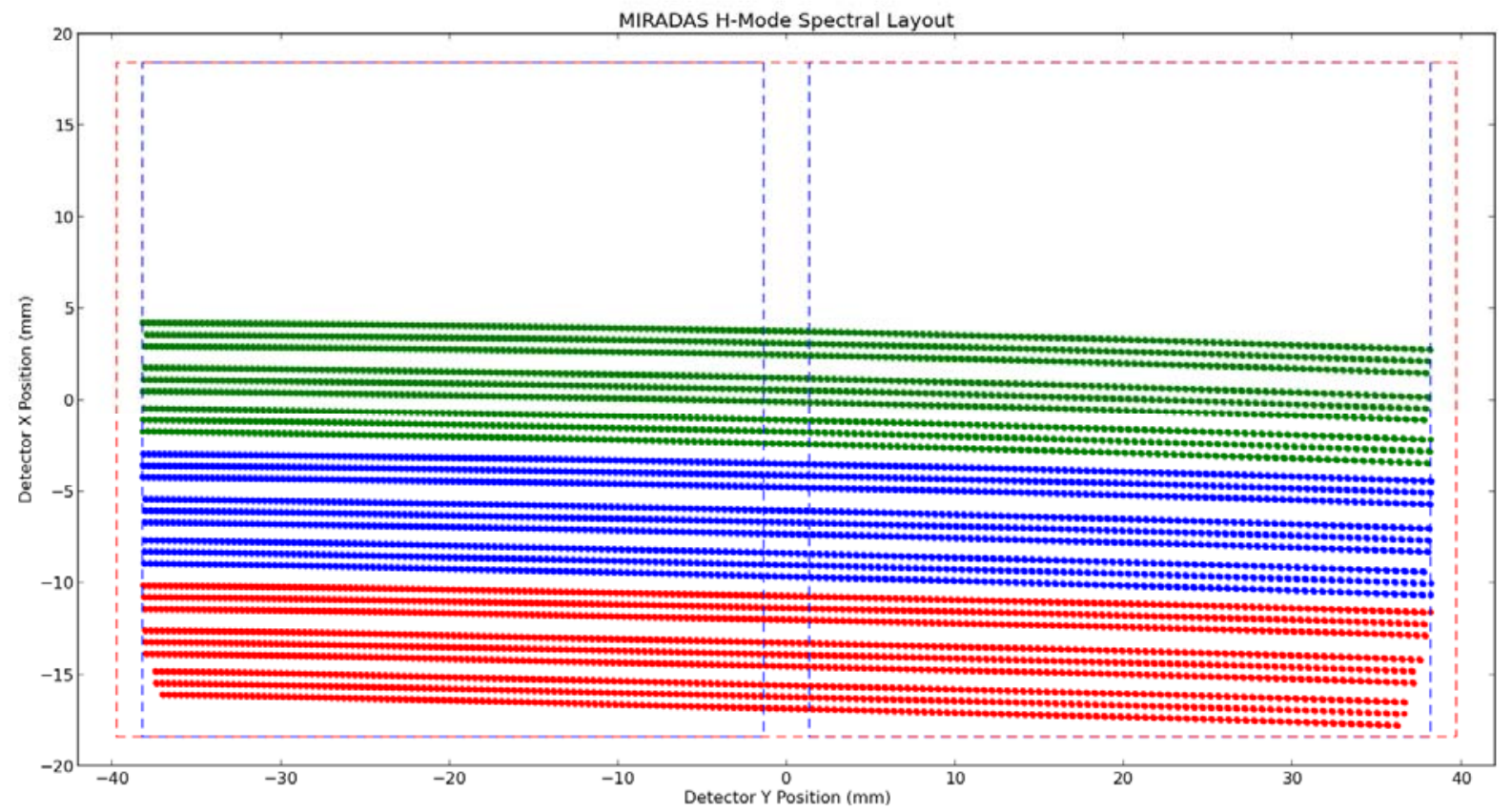

Figure 9 - MIRADAS medium-multiplex H-band mode cross-dispersed spectra projected on the 4096x2048-pixel detector mosaic. The spectrum covers the wavelength range from 1.54-1.76 $\mathrm{m}$ (orders 20-22) for 3 separate science targets simultaneously. 


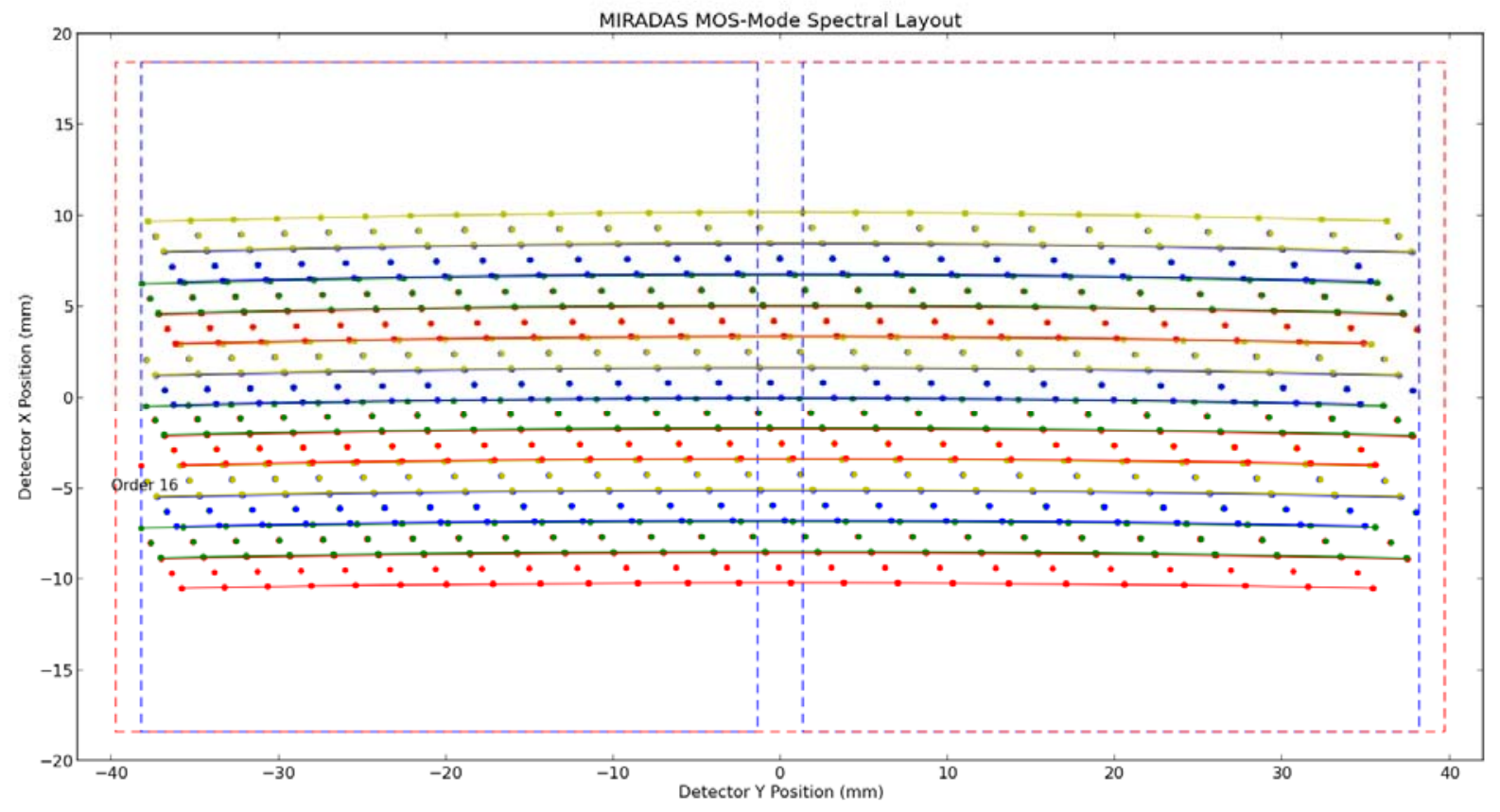

Figure 10 - MIRADAS maximum-multiplex mode spectra projected on the 4096x2048-pixel detector mosaic. The spectrum covers the wavelength range of any single order (order shown is 15) for 12 separate science targets simultaneously.

\section{DETECTOR ELECTRONICS AND DATA PIPELINE}

MIRADAS will use two Teledyne HAWAII-2RG arrays in a 4096x2048-pixel mosaic. For the Final Design we are using I-SDEC controllers (Ramaprakash et al., 2010) to interface with the FPA and ASIC. Control of each I-SDEC will handled by a single board computer (SBC) which are connected via USB. The SBC is an Adlink ReadyBoard 910 which is 166 x $115 \mathrm{~mm}$ in size, has an Intel I5 processor, and supports a Linux operating system. To minimize cabling between the ASIC and the I-SDEC the SBC and I-SDEC are mounted, in a custom aluminum enclosure, on the Dewar connector plate through which the flex cable is fed. Communication to the SBC will be through gigabit Ethernet. Maximum power required by each I-SDEC is $0.5 \mathrm{~A}$ at $5 \mathrm{~V}$, which is supplied by a linear power supply. Available power for the SBC is $4 \mathrm{~A}$ at $12 \mathrm{~V}$ which is also supplied by a linear power supply. Since the SBC power requirement is dependent on the amount of processing and on-board resources used by the software a larger power supply was chosen in order to not limit the software. Each I-SDEC and SBC has its own power supply that can be turned on or off remotely with the GTC MAD module. We show early test results from the ISDEC controller with a H2RG multiplexer in Figure 11. 


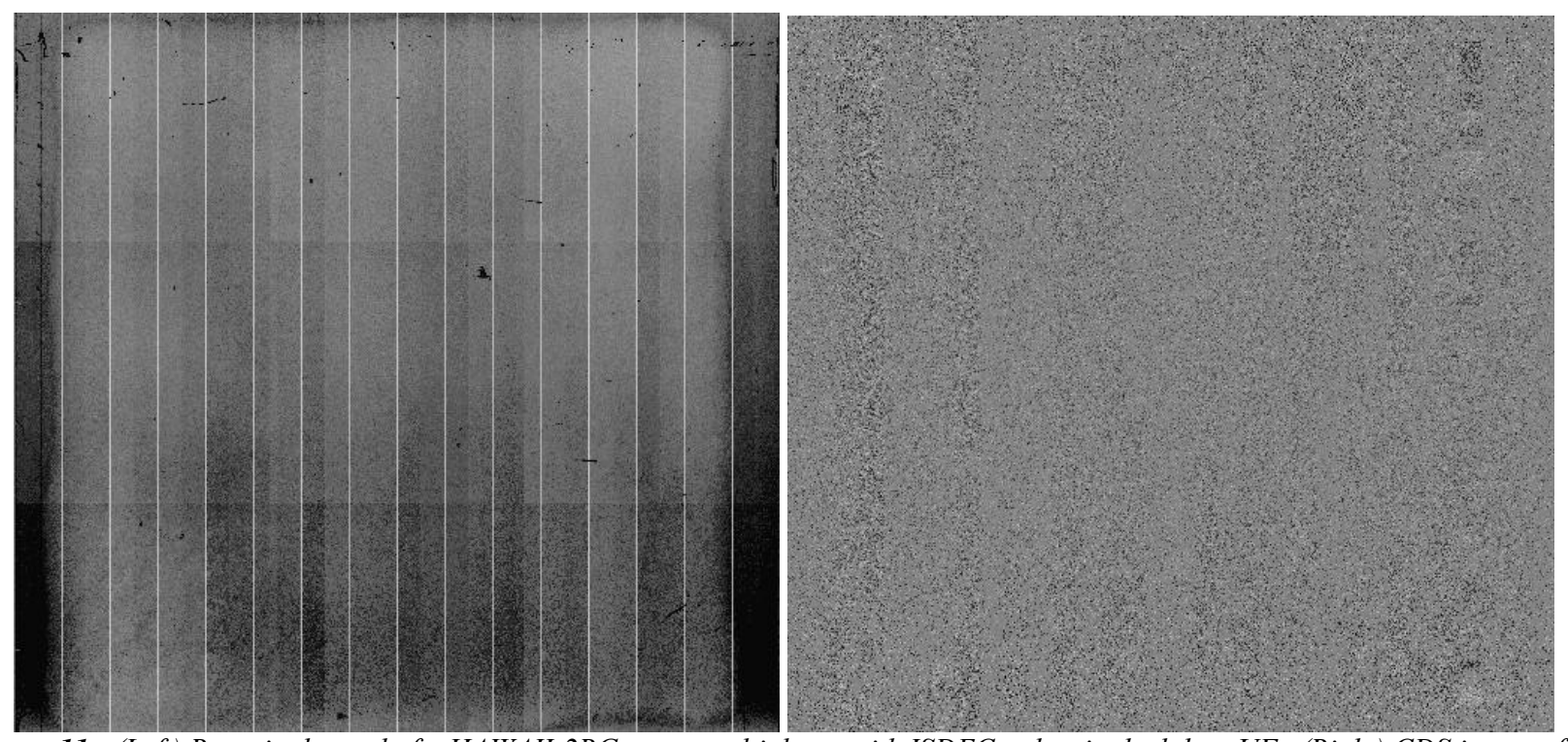

Figure 11 - (Left) Raw single read of a HAWAII-2RG warm multiplexer with ISDEC, taken in the lab at UF. (Right) CDS image of the same.

The MIRADAS data reduction pipeline is being developed at UCM, and is based on the Python pipeline experience there for previous GTC infrared instruments. The MIRADAS data pipeline will provide fully reduced and calibrated data for all modes of the instrument, including spectro-polarimetry.

\section{INSTRUMENT CRYOSTAT AND ENVELOPE}

The MIRADAS instrument conforms to the GTC envelope of mass/volume for the Folded Cassegrain focus. The protruding coldheads (Figure 12) require a variance, but do not interfere with other components/instruments on the telescope.
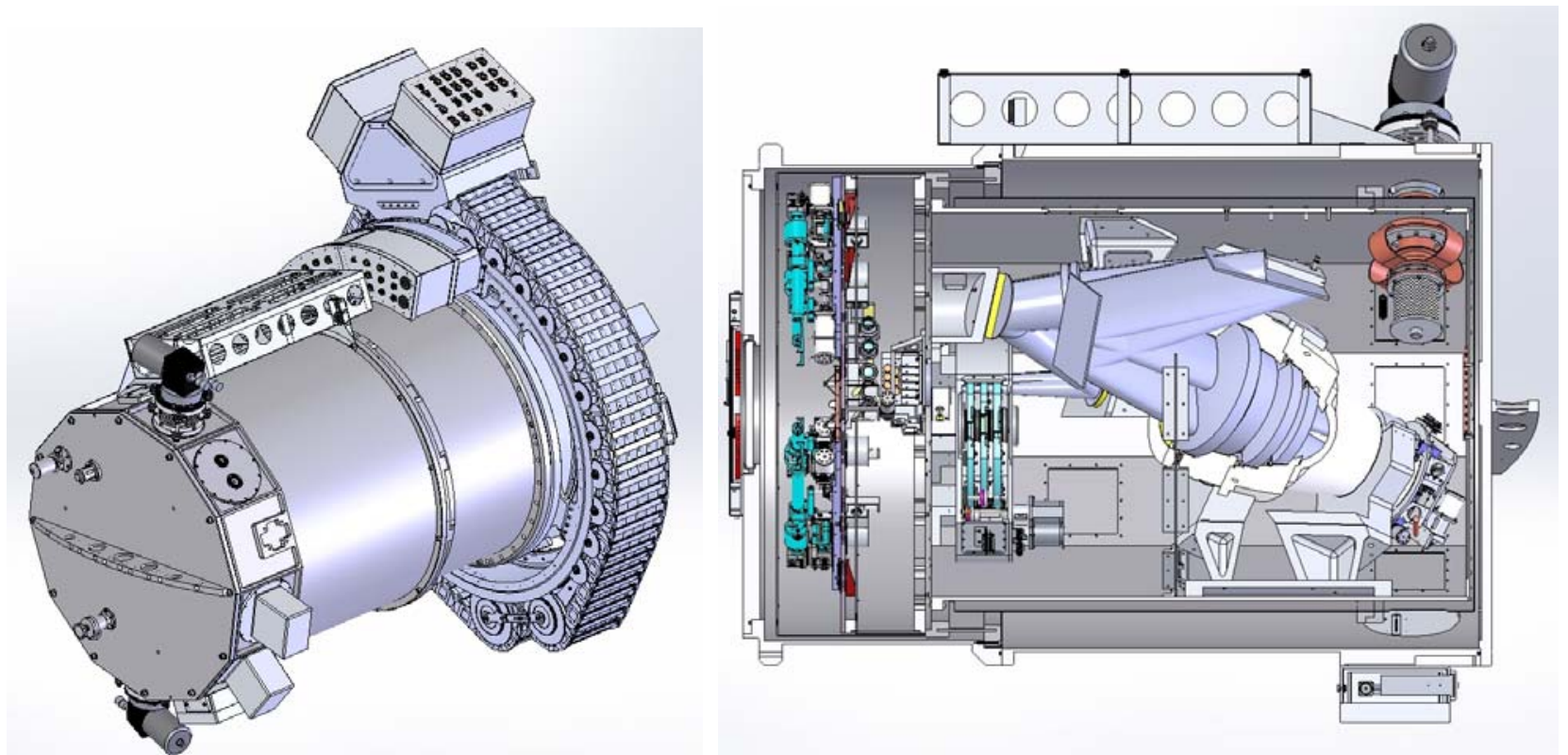

Figure 12 - MIRADAS instrument views. (Left) MIRADAS full instrument view installed on the GTC Folded Cassegrain rotator. (Right) Sectional view of MIRADAS. 


\section{FABRICATION UPDATES}

MIRADAS is currently in the early stages of its fabrication phase. We present some early photos of optical and mechanical elements, including the spectrograph camera being developed at NEOS, in the figures below.
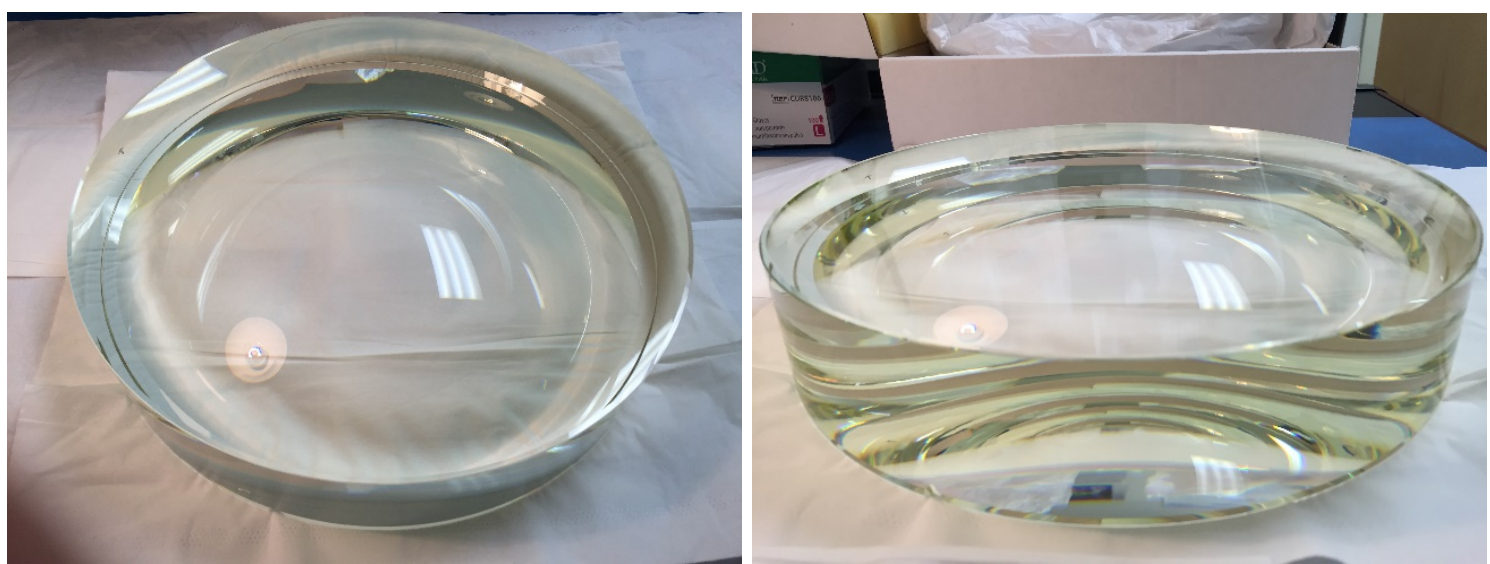

Figure 13 - MIRADAS OHARA S-FTM16 lens
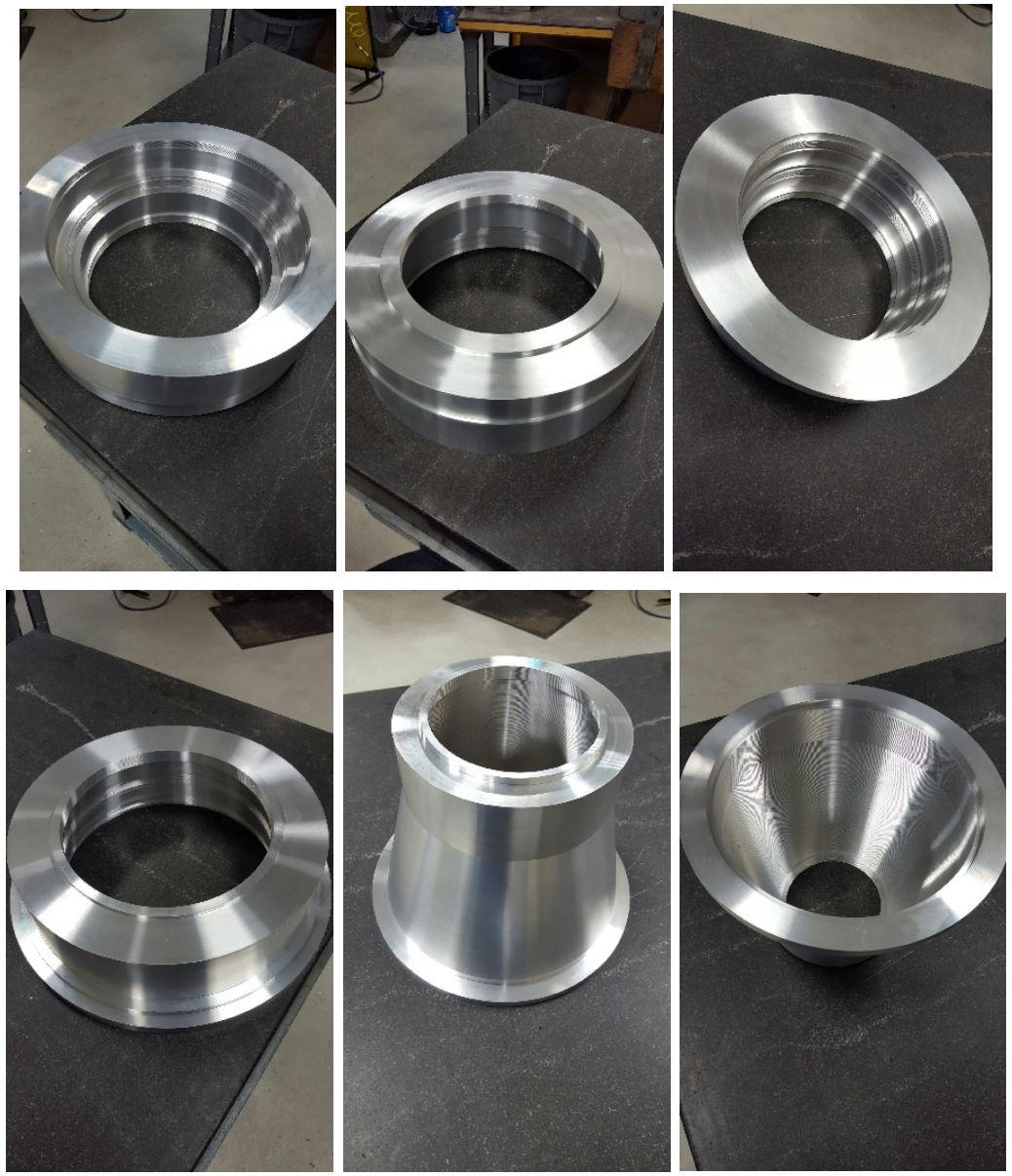

Figure 14 - MIRADAS Spectrograph camera housing 


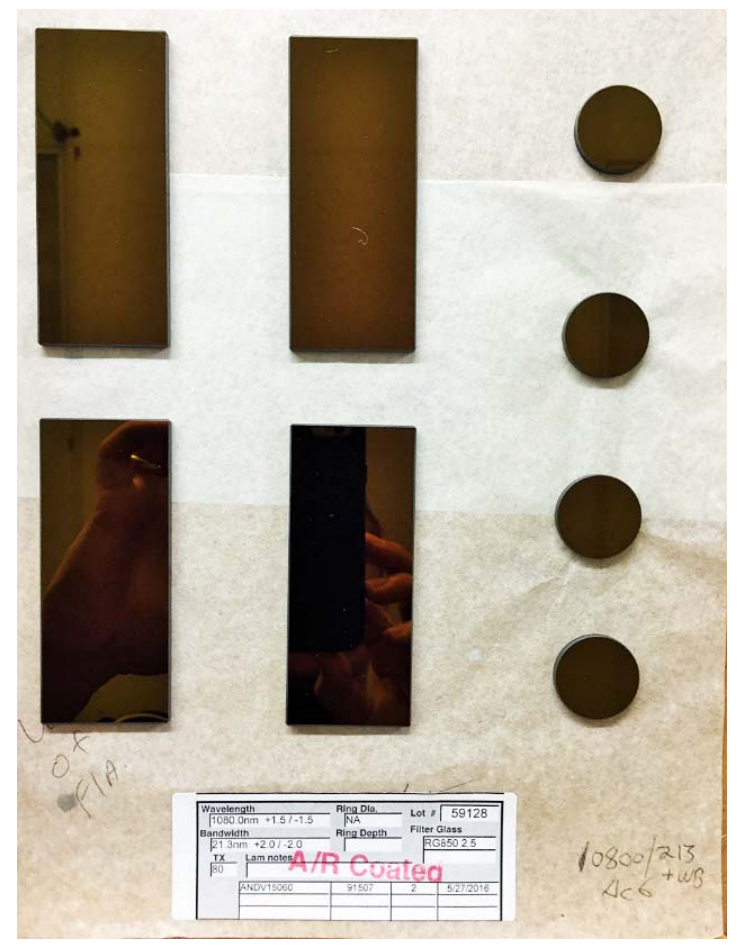

Figure 15 - MIRADAS Filters (J-lo \& H) at Andover
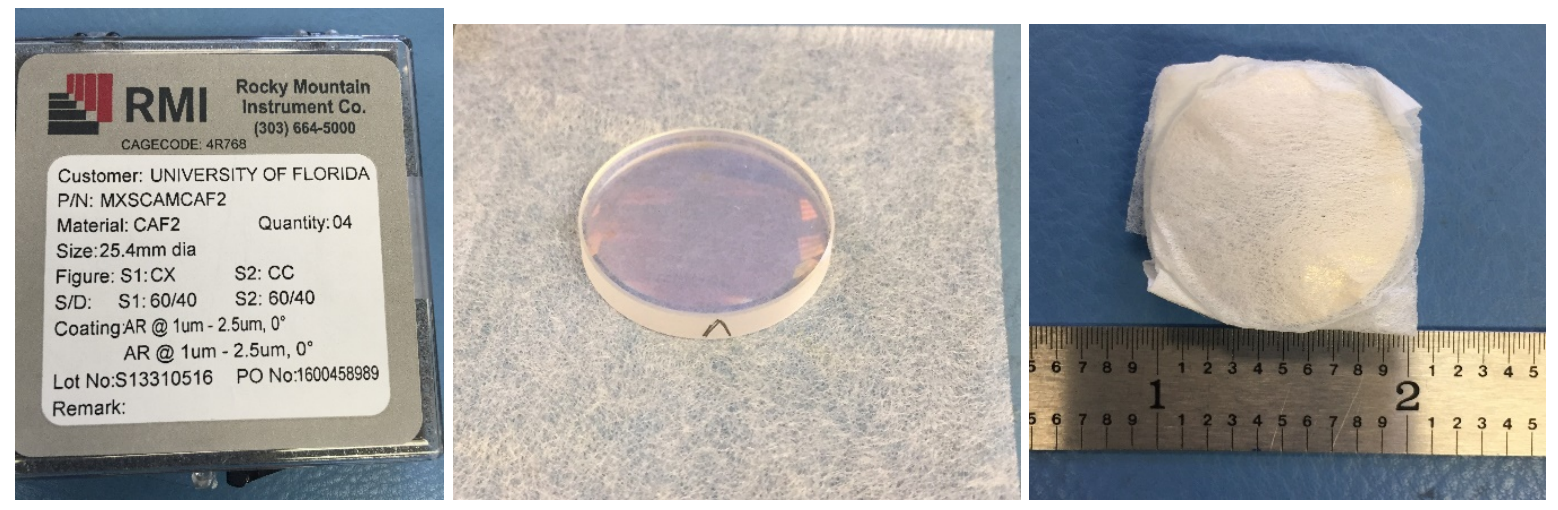

Figure 16 - MIRADAS MXS lenses

Proc. of SPIE Vol. 9908 99081L-15 


\section{REFERENCES}

Andersen, David R.; Eikenberry, Stephen S.; Fletcher, Murray; Gardhouse, William; Leckie, Brian; Véran, Jean-Pierre; Gavel, Don; Clare, Richard; Guzman, Rafael; Jolissaint, Laurent; Julian, Roger; Rambold, William, 2006, Proc. Of SPIE, Vol. 6269, 145

Eikenberry, Stephen S.; Murphey, Charles H.; Mullin, Scott A.; Bennett, John G.; Raines, Steven N.; Ackley, Kendall; Stelter, Richard D.; Garner, Alan; Sabater, Josep; Eikenberry, Sophia A.; Chinn, Brian; Donoso, Hipatia V.; Vega, Claudia S.; Gómez, José María.; Torra, Jordi; Herlevich, Michael D.; Frommeyer, Richard; Miller, Paola, 2014, Proc. Of SPIE, Vol. 9147, 0XE

Eikenberry, S. S.; Bennett, J. G.; Chinn, B.; Donoso, H. V.; Eikenberry, S. A.; Ettedgui, E.; Fletcher, A.; Frommeyer, Raymond; Garner, A.; Herlevich, M.; Lasso, N.; Miller, P.; Mullin, S.; Murphey, C.; Raines, S. N.; Packham, C.; Schofield, S.; Stelter, R. D.; Varosi, F.; Vega, C.; Warner, C.; Garzón, Francisco; Rosich, J.; Gomez, J. M.; Sabater, J.; Vilar, C.; Torra, J.; Gallego, J.; Cardiel, N.; Eliche, C.; Pascual, S.; Ballester, O.; Illa, J. M.; Jimenez, J.; Cardiel-Sas, L.; Galipienzo, J.; Carrera, M. A.; Hammersley, P.; Cuevas, S., 2012, Proc. Of SPIE, Vol. 8446, 57E

Eikenberry, Stephen; Hinkle, Kenneth; Joyce, Dick; Liang, Ming; Muller, Gary; Heileman, Ed; French, Jim; Ge, Jian; Packham, Chris; Julian, Roger; Gaughan, Neil; Sprayberry, David, 2006a, Proc. Of SPIE, Vol. 6271, 31E

Eikenberry, Stephen; Andersen, David; Guzman, Rafael; Bally, John; Cuevas, Salvador; Fletcher, Murray; Gardhouse, Rusty; Gavel, Don; Gonzalez, Anthony; Gruel, Nicolas; Hamann, Fred; Hamner, Sam; Julian, Roger; Julian, Jeff; Koo, David; Lada, Elizabeth; Leckie, Brian; Lopez, J. Alberto; Pello, Roser; Perez, Jorge; Rambold, William; Roman, Carlos; Sarajedini, Ata; Tan, Jonathan; Venn, Kim; Veran, Jean-Pierre; Ziegert, John, 2006b, Proc. Of SPIE, Vol. 6269, 188

Hinkle, Kenneth H.; Eikenberry, Stephen; Joyce, Richard; Liang, Ming; Muller, Gary; Hileman, Ed; French, Jim; Ge, Jian; Packham, Chris; Julian, Roger; Armandroff, Taft; Gaughan, Neil; Sprayberry, David, 2006, Proc. Of SPIE, Vol. 6269, 117

Liang, Ming; Joyce, R. R.; Eikenberry, S. S.; Hinkle, K. H.; Muller, G. P.; Ge, Jian; Sprayberry, David, 2006, Proc. Of SPIE, Vol. 6269, 139

Muller, G. P.; Hileman, E. A.; Hinkle, K. H.; Joyce, R. R.; Liang, M.; Sprayberry, D.; Eikenberry, S. S., 2006, Proc. Of SPIE, Vol. 6269, 142

Ramaprakash, Anamparambu; Burse, Mahesh; Chordia, Pravin; Chillal, Kalpesh; Kohok, Abhay; Mestry, Vilas; Punnadi, Sujit; Sinha, Sakya, 2010, Proc. of SPIE, Vol. 7742, 68

Sabater, Josep; Gómez, José María.; López, Manuel; Torra, Jordi; Raines, Steven N.; Eikenberry, Stephen S. 2014, Proc. of SPIE, 9151, 5SS

Sabater, Josep; Gómez, José María.; López, Manuel; Torra, Jordi; Raines, Steven N.; Eikenberry, Stephen S., 2016, these proceedings

Stelter, R.D. and Eikenberry, S.S., 2016, these proceedings. 